\section{Translation, adaptation and validation of Depression, Anxiety and Stress Scale in Urdu}

\author{
Waqar Husain ${ }^{1 *}$ and Amir Gulzar² \\ ${ }^{1}$ Assistant Professor of Psychology, Department of Humanities, COMSATS University Islamabad, \\ Pakistan \\ ${ }^{2}$ Head of Business \& Economics' Department, Foundation University Pakistan
}

\section{Abstract}

The current study was carried out to translate, adapt and validate Depression, Anxiety and Stress Scale in Urdu language and in Pakistani culture. The current translation was aimed to produce a more user friendly and clinically applied version of DASS. The systematic procedure involved in translation focused on maximizing levels of semantic and conceptual equivalence. A test re-test pilot study was conducted on 30 participants to analyze the translated version initially, the results of which determined a significant positive correlation between original and translated versions. The main study involved 300 conveniently selected Pakistanis. The findings revealed that the translated version titled U-DASS-42 was highly reliable and valid in Pakistani culture. The newly developed U-DASS-42 is attached with this paper for the convenience of Pakistani researchers.

\section{More Information}

*Address for Correspondence: Waqar Husain, Assistant Professor of Psychology, Department of Humanities, COMSATS University Islamabad, Pakistan, Tel: 923005960102 ; Email: drsukoon@gmail.com;

Submitted: 06 February 2020 Approved: 24 February 2020 Published: 25 February 2020

How to cite this article: Husain W, Gulzar A. Translation, adaptation and validation of Depression, Anxiety and Stress Scale in Urdu. Insights Depress Anxiety. 2020; 4: 001-004.

DOI: dx.doi.org/10.29328/journal.ida.1001011 ORCiD: orcid.org/0000-0003-3047-1834

Copyright: @ 2020 Husain W, et al. This is an open access article distributed under the Creative Commons Attribution License, which permits unrestricted use, distribution, and reproduction in any medium, provided the original work is properly cited.

Keywords: Depression; Anxiety; Stress; Scale; Urdu

Check for updates

OPen Access

\section{Introduction}

Stress, Anxiety \& Depression are widespread psychological problems. These are the most commonly diagnosed illnesses in Psychology [1,2-5]. Much consideration is given to these areas as the prevalence of these syndromes is increasing [6,7]. Several studies have associated these syndromes to numerous negative outcomes e.g. decreased work performance [8-10], general dissatisfaction [7,11], poor quality of life [12], and problems with social interactions [3,13]. Researchers have proposed that all populations, and particularly employees, are being affected by the aforesaid disturbances in epidemic proportions. Researchers [14-16] have suggested that each of these syndromes can be associated with decreased performance on the job, decreased satisfaction in the work environment, and poor interpersonal skills; which contribute to poor customer service, increased intention to leave, and impaired peer relations in the workplace.

Traditionally, it was believed that stress, anxiety and depression were separate constructs with varying etiologies and symptoms; however, the high co-morbidity rates, especially between anxiety and depression, have gained interest and attention throughout the field [17-20]. Some associations are also developed between stress and depression [21-23]. The development of more complex stress models has, however, provided support for a relationship between the syndromes [24].

Depression, Anxiety and Stress Scale [1] is a 42 items self reporting measure to assess prominent features of depression, anxiety and stress. The DASS was initially developed by Lovibond, 1995 [1] and since then it has been translated into many languages and in different cultures. The theoretical foundations of DASS are based on the tripartite model of depression and anxiety [25]. While stress being common in depression and anxiety, the model suggests that depression and anxiety have both common and uncommon features. Low positive affect and anhedonia, for example, are the unique depressive features. Physiological hyperarousal, on the other hand, is considered as a unique feature of anxiety. The same model has been supported in several studies around the globe e.g. Watson, Clark, Weber, Assenheimer, Strauss and McCormick, [26] and Endler, Macrodimitris, and Kocovski [27]. The DASS has been considered a highly reliable scale with its distinguishing features and many studies have found its subscales highly reliable in clinical settings [2,28-31]. As the scale is heavily used by the students of social sciences in Pakistan, the current study was therefore aimed at translating DASS in a maximized user friendly fashion and to further adapt and validate the scale in Pakistani context. 


\section{Method}

\section{Translation and adaptation}

The procedure adapted to translate DASS in Urdu involved maximized levels of semantic and conceptual equivalence [32] between the original English and translated Urdu versions of DASS. Semantic equivalence refers to the literal similarity between original and translated versions, whereby the conceptual equivalence is related to the cultural adaptability of the translated version (Figure 1).

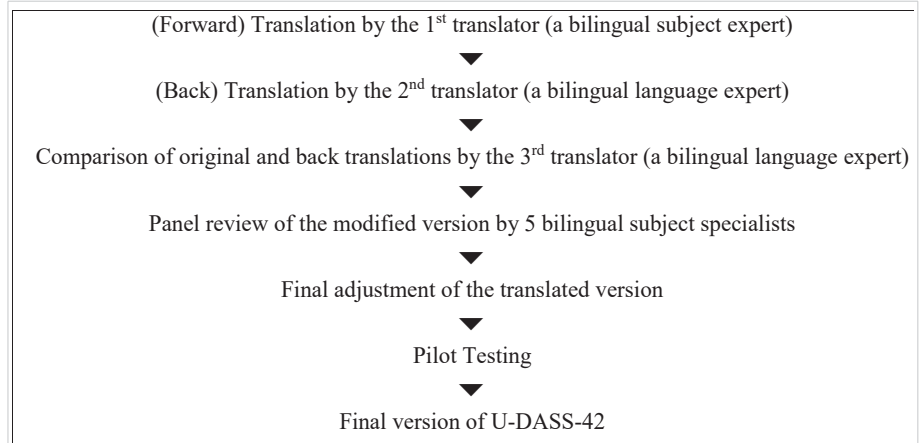

Figure 1: Steps involved in the process of translation and adaptation.

The original version of DASS was (forward) translated into Urdu by a bilingual Clinical Psychologist in the Department of Psychology, Foundation University Pakistan (1 $1^{\text {st }}$ author). The translated version was back-translated into English by a professional translator of the Department of English of the same university. The translator was unfamiliar with the original English version of DASS. Both the back-translated English version and the original English version of DASS was examined by another professional translator of the same department who thereafter, with the help of the bilingual Clinical Psychologist involved, adjusted the translated version to maximize levels of semantic and conceptual equivalence so that the translated version could be best adapted in Urdu language and in Pakistani culture. A panel comprising of 5 Psychology teachers was constituted thereafter to provide their feedback on the translated version. The modifications proposed by the panel were incorporated accordingly. The final translated version of DASS was piloted with 30 students of the same university. Two groups, each comprising of 15 randomly selected participants, were made in this regard. Group A was given the original English version of DASS and Group B was provided with the translated version. Their scores were analyzed and recorded. The same groups were re-tested two weeks later than the first test whereby Group A was now given the translated version and Group B was given the original DASS in English. The correlation between the two versions for Group A was .72 and .70 for Group B which concluded that both the versions are positively correlated with each other on highly significant levels.

These finding also revealed that the Pakistani participants easily attempted both versions of DASS and had no linguistic problems in this regard. Moreover, all the items of the translated version were understandable in Pakistani culture. The final translated items are presented in table 1. After translation, a modification was carried out in the item numbering of the translated scale to make it easier for the users to administer any of the sub-scale separately instead of finding which item is of depression, anxiety or stress. The translated version is produced in such a sequence whereas items 1 to 14 are of depression, items 15 to 28 are of anxiety, and items 29 to 42 are of stress. The sequence and proper placement of probing questions in each scale was also given appropriate consideration in this regard as projected in table 1 . Another distinguished feature of the current translated version of DASS is its maximized user-friendliness. The condition of "last week" for the prevalence of each symptom as desired by the original DASS in English is included in every translated item so that the respondents would not forget that the statements are about the last week only. Furthermore, in the response section, the four possible choices are kept visible in front of each statement so that the respondents could easily place a tick on the relevant response without being confused in 0 to 3 rating. The title for the translated version is U-DASS- 42 i.e. Urdu version of DASS comprising of 42 items. The U-DASS-42 can be viewed further as annexed to this paper. Translation and re-numbering of items is reflected in the table 1 (see in Annex File).

\section{Scoring}

The scoring of U-DASS-42 is the same as of its original English version by Lovibond \& Lovibond [1]. The sum of the scores of each scale is calculated and analyzed for its severity on the "severity rating index" as provided by Lovibond \& Lovibond [1] (Table 2).

\begin{tabular}{|c|c|c|c|}
\hline \multicolumn{4}{|c|}{ Table 2: Severity Rating Index of Depression, Anxiety, and Stress Scale. } \\
\hline & Scoring and Interpretation of DASS \\
\hline Normal & $0-9$ & $0-7$ & $0-14$ \\
\hline Mild & $10-13$ & $8-9$ & $15-18$ \\
\hline Moderate & $14-20$ & $10-14$ & $19-25$ \\
\hline Severe & $21-27$ & $15-19$ & $26-33$ \\
\hline Extremely Severe & $28+$ & $20+$ & $34+$ \\
\hline
\end{tabular}

\section{Method}

\section{Participants}

In addition to the pilot study conducted on 30 participants, the sample of the main study comprised of 300 conveniently selected participants comprising of both sexes i.e. 150 males and 150 females. All the participants of the study were adults residing in Rawalpindi and Islamabad, Pakistan. Their ages ranged between 18 and 49 .

\section{Procedure}

The participants of the study were contacted by the researchers by visiting different academic institutions and 
public sector organizations. The issues of confidentiality and compliance to the ethical standards were given proper consideration. The data obtained was analyzed using the Statistical Package for Social Sciences (SPSS).

\section{Results}

\section{Reliability and construct validity of U-DASS-42}

The U-DASS-42 and its subscales were found highly reliable and valid (Table 3 ).

\section{Table 3: Reliability and Construct Validity of U-DASS-42.}

\begin{tabular}{|c|c|c|c|c|c|c|c|} 
Scale & M & SD & a & U-DASS-42 & Depression & Anxiety & Stress \\
\hline U-DASS-42 & 2.17 & 1.50 & .91 & - & $.909^{\star \star}$ & $.973^{\star *}$ & $.917^{\star \star}$ \\
\hline Depression & 2.44 & 1.24 & .86 & & - & $.841^{\star *}$ & $.690^{\star \star}$ \\
\hline Anxiety & 2.11 & 1.16 & .74 & & & - & $.887^{\star \star}$ \\
\hline Stress & 2.58 & 1.14 & .86 & & & & - \\
\hline
\end{tabular}

With reference to table 3, the Urdu version of Depression, Anxiety and Stress Scale (U-DASS-42) was found highly reliable by measuring its reliability on Cronbach's alpha. The internal consistency reliability coefficients for the overall scale and its subscales for depression, anxiety and stress were $.91, .86, .74$ and .86 respectively. The analysis for construct validity of U-DASS-42 revealed significant positive correlation between U-DASS-42 and its subscales i.e. depression $(r=.91, p$ $<.001)$ anxiety $(r=.97, p<.005)$, and stress $(r=.92, p<.001)$ (Table 4) (see in Annex File).

The above table shows total item correlation of U-DASS-42 and the correlation of each of the subscale's item with the subscale. The correlation is significant at 0.001 levels (Table 5) (see in Annex File).

Table 5 projects the convergent validity of U-DASS- 42 . The values of Square Multiple Correlations (SMCs) being equal to or greater than 0.6 along with path coefficients equal to or greater than 0.7 reveal strong convergent validity of U-DASS-42 (Tables 6,7).

Table 6: Discriminant Validity of U-DASS-42.

\begin{tabular}{|c|c|c|c|}
\hline & Depression & Anxiety & Stress \\
\hline Depression & 1 & & \\
\hline Anxiety & .811 & 1 & \\
\hline Stress & .638 & .872 & 1 \\
\hline
\end{tabular}

Table 7: Construct Validity: Exploratory Factor Analysis of U-DASS-42.

\begin{tabular}{|c|c|c|c|c|c|}
\hline $\begin{array}{c}\text { Latent } \\
\text { Variables }\end{array}$ & DCM $^{\mathbf{a}}$ & $\mathbf{K M O}^{\mathbf{b}}$ & BTS $^{\mathbf{X}} \mathbf{X}^{2}$ & $\begin{array}{c}\text { Component } \\
\text { Extracted }\end{array}$ & $\begin{array}{c}\text { Variance Explained } \\
(\%)\end{array}$ \\
\hline Depression & .006 & .644 & $1515^{\star}$ & 1 & 71 \\
\hline Anxiety & .022 & .714 & $1122^{\star}$ & 1 & 81 \\
\hline Stress & .012 & .738 & $2688^{*}$ & 1 & 86 \\
\hline
\end{tabular}

a Determination of Correlation Matrix; ${ }^{b}$ Kaiser-Meyer-Olkin Measure of Sample Adequacy; 'Bartlett's Test of Sphericity; ${ }^{*} p<.001$

\section{Discussion and conclusion}

The current study was carried out to translate, adapt and validate Depression, Anxiety and Stress Scale [1] in Urdu language and in Pakistani culture. The original scale (English) has been widely used and translated into several languages. The scale was already translated in Urdu before by four different researchers; hence those translations were carried out for academic purposes. The current translation was aimed to produce a more user friendly and clinically applied version of DASS. The procedure involved in translation focused on maximized levels of semantic and conceptual equivalence. A test re test pilot study was conducted on 30 participants to analyze the translated version initially, the results of which determined a significant positive correlation between the original and translated versions. The main study involved 300 conveniently selected Pakistanis. The findings revealed the translated version titled U-DASS- 42 was highly reliable and valid in Pakistani culture. The internal consistency reliability coefficients for the overall scale and its subscales for depression, anxiety and stress were $0.91,0.86,0.74$ and .86 respectively. The analysis for construct validity of U-DASS-42 revealed significant positive correlation between U-DASS-42 and its subscales i.e. depression $(r=0.91, p<0.001)$ anxiety $(r=0.97, p<0.05)$, and stress $(r=0.92, p<0.001)$. The totalitem and item-scale reliability was also analyzed and found highly reliable. The values of Square Multiple Correlations (SMCs) revealed strong convergent validity of U-DASS-42. The correlation between latent variables confirmed the discriminant validity of the scale. The determinant values of the entire constructs revealed no multicollinearity and singularity issues proving a reliable construct validity of the scale.

The newly developed U-DASS-42 is hereby presented free of charge for the convenience of Pakistani researchers and mental health practitioners. The instrument can be used for clinical diagnosis and as well as for the purpose of research.

Tables 1,4,5 and Annex A File

\section{References}

1. Lovibond PF, Lovibond SH. The structure of negative emotional states: Comparison of the Depression Anxiety Stress Scales (DASS) with the Beck Depression and Anxiety Inventories. Behav Res Ther. 1995; 33: 335-343.

PubMed:https://www.ncbi.nlm.nih.gov/pubmed/7726811

2. Brown TA, Chorpita BF, Korotitsch W, Barlow DH. Psychometric properties of the Depression Anxiety Stress Scales (DASS) in clinical samples. Behav Res Ther. 1997; 35: 79-89.

PubMed:https://www.ncbi.nlm.nih.gov/pubmed/9009048

3. Davies F, Norman R, Cortese L, Malla A. The relationship between types of anxiety and depression. J Nervous Mental Disease. 1995; 183: 31-35.

4. Farmer RF. Depressive symptoms as a function of trait anxiety and impulsivity. J Clin Psychol. 1998; 54: 129-135.

PubMed:https://www.ncbi.nlm.nih.gov/pubmed/9467756

5. Ollendick T, Yule W. Depression in British and American children and its relation to anxiety and fear. J Consult Clin Psychol. 1990; 58: 126-129. PubMed:https://www.ncbi.nlm.nih.gov/pubmed/2319046

6. Cyranowski J, Frank E, Young E, Shear K. Adolescent onset of the 
gender difference in lifetime rates of major depression. Arch Gen Psychiatry. 2000; 57: 21-27.

PubMed:https://www.ncbi.nlm.nih.gov/pubmed/10632229

7. Kessler RC, McGonagle KA, Zhao S, Nelson CB, Hughes $M$, et al Lifetime and 12-month prevalence of DSM-III-R psychiatric disorders in the United States: Results from the National Comorbidity Study. Arch Gen Psychiatry. 1994; 51: 8-19.

PubMed:https://www.ncbi.nlm.nih.gov/pubmed/8279933

8. Greenberg J. Comprehensive stress management. Dubuque, IA: WCB McGraw-Hill. 1999.

9. Poole W. The art of healing. Atlanta, GA: Turner. 1993.

10. Seaward B. Managing stress: Principles and strategies for health and well-being. Sudbury, MA: Jones and Bartlett. 1999.

11. Youngren $M$, Lewinsohn $P$. The functional relationship between depression and problematic interpersonal behavior. J Abnorm Psychol. 1980; 89: 333-341.

PubMed:https://www.ncbi.nlm.nih.gov/pubmed/7410700

12. Norvell $\mathrm{N}$, Hills $\mathrm{H}$, Murrin $\mathrm{M}$. Understanding stress in female and male law enforcement officers. Psychology of Women Quarterly. 1993; 17: 289-301.

13. Alden $\mathrm{L}$, Phillips N. An interpersonal analysis of social anxiety and depression. Cognitive Therapy and Research. 1990; 14: 499-513.

14. Geller P, Hobfoll S. Gender differences in job stress, tedium and socia support in the workplace. J Social and Personal Relationships. 1994; 11: $555-572$

15. Eaton WW, Kessler RC, Wittchen HU, Magee WJ. Panic and panic disorder in the United States. Am J Psychiatry. 1994; 151: 413-420. PubMed:https://www.ncbi.nlm.nih.gov/pubmed/8109651

16. Lovibond P. Long-term stability of depression, anxiety and stress syndromes. J Abnorm Psychol. 1998; 107: 520-526.

PubMed:https://www.ncbi.nlm.nih.gov/pubmed/9715586

17. Lovibond S, Lovibond P. Manual for the Depression Anxiety Stress Scales (2nded.).Sydney, Australia: Psychology Foundation. 1995.

18. Stavrakaki C, Vargo B. The relationship of anxiety and depression: A review of the literature. $\mathrm{Br} J$ Psychiatry. 1986; 149: 7-16. PubMed:https://www.ncbi.nlm.nih.gov/pubmed/3535981

19. Dobson K. The relationship between anxiety and depression. Clinical Psychology Review. 1985; 5: 307-324.

20. Feldman L. Distinguishing depression and anxiety in self-report: Evidence fromconfirmatory factor analysis on nonclinical and clinical samples. J Consult Clin Psychol. 1993; 61: 631-638.

PubMed:https://www.ncbi.nlm.nih.gov/pubmed/8370858
21. Hammen CL. The generation of stress in the course of unipolar depression. J Abnorm Psychol. 1991; 100: 555-561. PubMed:https://www.ncbi.nlm.nih.gov/pubmed/1757669

22. Kirkcaldy B, Cooper C, Ruffalo P. Work stress and health in a sample of U.S. police. Psychol Rep. 1995; 76: 700-702. PubMed:https://www.ncbi.nlm.nih.gov/pubmed/7667485

23. Lazarus R. Puzzles in the study of daily hassles. J Behav Med. 1984; 7 : 375-389.

PubMed:https://www.ncbi.nlm.nih.gov/pubmed/6520869

24. Davila J, Hammen C, Burge D, Paley B, Daley S. Poor interpersonal problem solving as a mechanism of stress generation in depression among adolescent women. J Abnorm Psychol. 1995; 104: 592-600. PubMed:https://www.ncbi.nlm.nih.gov/pubmed/8530761

25. Clark LA, Watson D. Tripartite model of anxiety and depression: Psychometric evidence and taxonomic implications. J Abnorm Psychol. 1991; 100: 316-336.

PubMed:https://www.ncbi.nlm.nih.gov/pubmed/1918611

26. Watson D, Clark LA, Weber K, Assenheimer JS, Strauss ME, et al. Testing a tripartite model: I. Evaluating the convergent and discriminant validity of anxiety and depression symptom scales. J Abnorm Psychol. 1995; 104: 3-14.

PubMed:https://www.ncbi.nlm.nih.gov/pubmed/7897050

27. Endler NS, Macrodimitris SD, Kocovski NL. Anxiety and depression: Congruent, separate, or both? J Applied Biobehavioural Research. 2003; 8: 42-60.

28. Antony MM, Bieling PJ, Cox BJ, Enns MW, Swinson RP. Psychometric properties of the 42-item and 21-item versions of Depression Anxiety Stress Scales in clinical groups and a community sample. Psychological Assessment. 1998; 10: 176-181.

29. Clara IP, Cox BJ, Enns MW. Confirmatory factor analysis of the Depression Anxiety Stress Scales in depressed and anxious patients. $J$ Psychopathol Beheavior. 2001; 23: 61-67.

30. Crawford JR, Henry JD. The Depression Anxiety Stress Scales (DASS): Normative data and latent structure in a large non-clinical sample. $\mathrm{Br} \mathrm{J}$ Clin Psychol. 2003; 42: 111-131.

PubMed:https://www.ncbi.nlm.nih.gov/pubmed/12828802

31. Nieuwenhuijsen $\mathrm{K}$, de Boer AG, Verbeek JH, Blonk RW, van Dijk FJ. The Depression Anxiety Stress Scales (DASS): Detecting anxiety disorder and depression in employee absent from work because of mental health problems. Occup Environ Med. 2003; 60: 177-182. PubMed:https://www.ncbi.nlm.nih.gov/pubmed/12782751

32. Flaherty JA, Gaviria FM, Pathak D, Mitchell T, Wintrob $R$, et al. Developing instruments for cross-cultural psychiatric research. J Nerv Ment Dis. 1988; 176: 257-263.

PubMed:https://www.ncbi.nlm.nih.gov/pubmed/3367140 